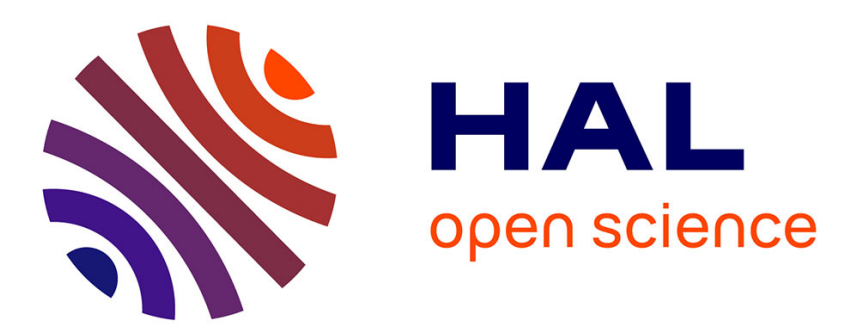

\title{
Robust OFDM Modem for a Swarm of Underwater Autonomous Vehicles
}

\author{
Nicolas Grollier, Guillaume Ansel, Christophe Laot
}

\section{To cite this version:}

Nicolas Grollier, Guillaume Ansel, Christophe Laot. Robust OFDM Modem for a Swarm of Underwater Autonomous Vehicles. IEEE Oceans 2019, Jun 2019, Marseille, France. 10.1109/OCEANSE.2019.8867069 . hal-02166680

\section{HAL Id: hal-02166680 https://hal.science/hal-02166680}

Submitted on 27 Jun 2019

HAL is a multi-disciplinary open access archive for the deposit and dissemination of scientific research documents, whether they are published or not. The documents may come from teaching and research institutions in France or abroad, or from public or private research centers.
L'archive ouverte pluridisciplinaire HAL, est destinée au dépôt et à la diffusion de documents scientifiques de niveau recherche, publiés ou non, émanant des établissements d'enseignement et de recherche français ou étrangers, des laboratoires publics ou privés. 


\title{
Robust OFDM Modem for a Swarm of Underwater Autonomous Vehicles
}

\author{
Nicolas Grollier, Guillaume Ansel, Christophe Laot \\ IMT Atlantique, Lab-STICC UMR CNRS 6285, UBL, 29238 Brest, France \\ Email: firstname.lastname@imt-atlantique.fr
}

\begin{abstract}
In this article, a filter bank is coupled with a CFAR detector to guarantee an efficient frame detection in presence of Doppler shift. This work is realized in scope of the NEMOSENS project, which aims to produce autonomous underwater vehicles (AUVs) able to communicate and move in a network thanks to UA modems. Many adverse phenomenons occur in the context of underwater acoustic. Most harmful effects for underwater acoustic communication (UAC) are the multi-path nature of the environment, the Doppler spread and the noise variability. The proposed method reduces the number of lost frames and gives a rough estimate of delay and Doppler shift. The followed approach is supported by simulations with simplified hypotheses, but the interest of this approach is also shown in real sea experiments.
\end{abstract}

Index terms - underwater acoustics communications, Doppler shift, synchronization

\section{INTRODUCTION}

The networking of heterogeneous underwater devices as autonomous underwater vehicles (AUV) or underwater positioning systems becomes more present with the deployment of reliable underwater acoustics (UA) communication techniques. Such a technology may be used for scientific [1] or industrial [2] purposes. In both cases, a reliable communication between nodes is mandatory. The communication function is assumed by an UA modem, that is a key component of the whole system.

In this paper, a part of the modem that is the basis of the NEMOSENS project is presented. The NEMOSENS project aims to develop a swarm of low cost AUVs, able to change their modem parameters to improve the communication reliability within the network. NEMOSENS is an officialy recognized project of the global economic competitiveness
"Pôle Mer Bretagne", funded by BPI France and Region Bretagne. RTsys is the work package leader with the partnership of FMC, ENSTA Bretagne and IMT-Atlantique [3]. A network where each AUV is a node of equal importance is considered, which indicates that there is no central network administration. A robust TDMA with guard interval as the MAC management method is used to show the proof-of-concept. To save useful transmission time, the system is designed to have no acknowledgment mechanism accepting frame lost.

In this paper a focus is made on the frame detection of the UWA modem. An orthogonal division multiplexing (OFDM) scheme is used, as it is known for its efficiency. To decrease the channel effect, cyclic prefixes and a root raised cosine as a pulse shaping function defined in the time domain are defined. The full description of the modem is developed in [4].

A locking mode performs the detection and synchronization with the received signal, which is highly degraded due to effects of the medium. This topic is often forgotten in applications but is essential to the detection of incoming frames. Among other degradation, the received signal is affected by a time dilation due to a Doppler shift as described in [5]. The article [5] is a reference of the state of the art and consists to use linear frequency modulated (LFM) signals around the transmitted frame. By comparing the delay between the LFM signals at the receiver with the expected delay, it becomes possible to estimate the Doppler and compensate it by interpolation. As a result, the received signal is compressed or expanded in time. Nonetheless, in [5] the Doppler shift is assumed constant over the frame, which is far from the ground truth if the frame 
duration is important.

The approach of [6] where a matched filter bank is used seems also interesting. It relies on a preamble presence, constituted by two identical OFDM symbols preceded by a cyclic prefix. In [6], it is proposed to correlates the received signal with delayed and re-sampled versions of itself in a parallel strategy. The [6] method is interesting as it maximizes the signal to noise ratio, and could be used to detect frames, estimate the Doppler shift and perform the synchronization. But, it requires an assumption of a constant Doppler shift over two OFDM symbols period.

It is proposed here to synchronize received frame with only one OFDM pilot symbol. In its original version [4], the locking mode was made thanks to an analysis of the spatial coherence between sub-carriers. It appears in practice that this estimation is efficient, but can be improved. In this paper, a change of the locking mode mechanism of [4] is proposed following the work in [6]. Nonetheless, the receiver's knowledge of the pilot symbol is used here instead of sending two identical OFDM symbols. Each filter is therefore a dilated and delayed version of the first symbol of the frame. So, the filter bank output global maximum represents the highest correlation with a particular version of the pilot symbol. In presence of a communication signal, the coordinates of the global maximum gives the beginning of the frame and an estimate of the Doppler shift. The output of the matched filter bank is a function of delay and Doppler shifts. Switching into decoding mode will be effective if the global maximum of the highest correlation is greater than a detection threshold.

This paper focuses on a method to obtain an adaptive threshold in function of the signal to noise ratio (SNR) variations. Originally used in a RADAR context [7], a CA-CFAR detector is of a particular interest here. Its input will be the output of the matched filter that gives the maximum correlation. The CA-CFAR output gives an adaptive detection threshold that can be compared to the filter bank output, and make a decision. A well-chosen set of CACFAR parameters make the whole system more robust to SNR and channel variations. In the following paper, the matched filter bank configuration is presented with a method that allows to adapt its parameters to the signal characteristics. Then, an analysis of the CACFAR configuration based on computer simulations is made. Thirdly, a validation is made on a sea record database.

\section{System Model}

\section{A. Transmitter Model}

Let first consider the OFDM transmitter. The subcarrier spacing $B=1 / T_{0}$ is proportional to the OFDM symbol duration $T_{0}$. Let $T=T_{0}+\tau$ the nominal duration of each OFDM symbol, where $\tau$ is the cyclic prefix (CP) duration. As proposed in [4], the transmitted signal model is written as:

$$
s(t)=\sum_{k=0}^{\infty} \sum_{n=0}^{N-1} d_{k, n} g(t-k T) e^{\jmath 2 \pi f_{n} t}
$$

where $N$ is the number of OFDM subcarrier, $d_{k, n}$ is a DQPSK (differential QPSK) information symbol transmitted on $f_{n}$, the $n^{\text {th }}$ subcarrier and $g(t-k T)$ is a root raised cosine pulse shaping function defined by:

$$
g(t)=\sqrt{B} \cdot \begin{cases}0 & \text { if } t<0, \\ \sin \left(\frac{\pi}{2} \frac{B}{\alpha} t\right) & \text { if } 0 \leq t<\frac{\alpha}{B} \\ 1 & \text { if } \frac{\alpha}{B} \leq t<\frac{1}{B} \\ \sin \left(\frac{\pi}{2} \frac{B}{\alpha}(T-t)\right) & \text { if } 0 \leq t<\frac{\alpha}{B} \\ 0 & \text { if } t \geq T .\end{cases}
$$

In (2), $\alpha$ stands for the a roll-off factor and belongs to $[0,1]$. The shaping filter operation decreases the energy at the edges of OFDM symbols. As a main benefit, it mitigates the energy of inter symbol interference (ISI), as developed in [8]. For our modem the shaping function $g(t)$ is used both at transmission and reception.

The DQPSK modulation is used to avoid channel estimation and equalization. In addition, to make a more robust transmission a convolution code and a bit interleaver are used.

\section{B. Receiver Model}

The received signal $y(t)$ is assumed affected by two adverse perturbations: a delay $t_{0}$ and a Doppler dilatation $D=\left(1-\nu_{0} / c\right)$ (with $\nu_{0}$ the relative speed between transmitter/receiver, and $c$ the acoustic propagation speed in water) such that:

$$
y(t, D)=s\left(D\left(t-t_{0}\right)\right)+w(t)
$$




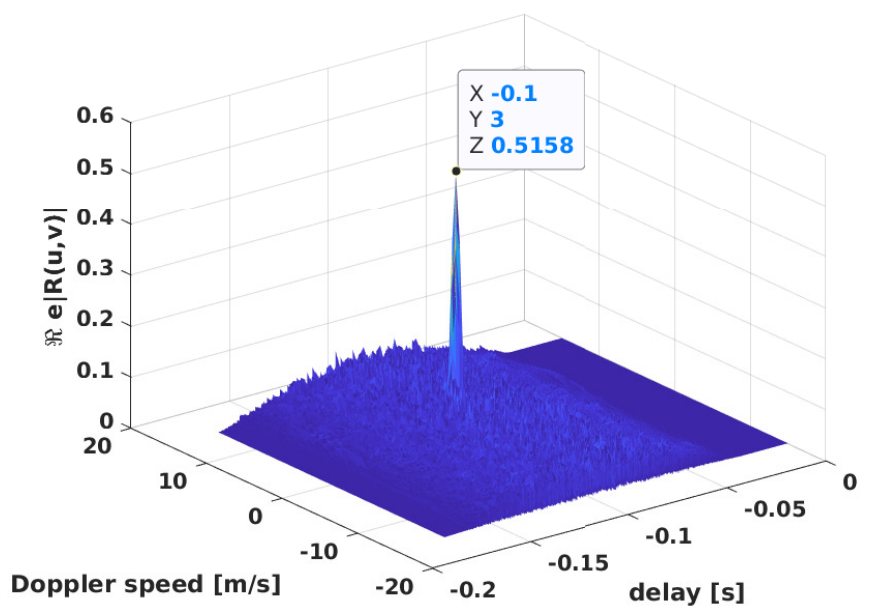

Fig. 1. Filter bank output, Doppler speed $=3 \mathrm{~m} / \mathrm{s}$, delay $t_{0}=$ $0.1 \mathrm{~s}, \mathrm{SNR}=0 \mathrm{~dB}$

An AWGN channel $w(t)$ of variance $\sigma_{w}^{2}$ is considered to develop our analysis. The UA noise is usually not white, but an analysis with simplified assumptions enables a validation before further experiments at sea. Moreover, in realistic UWA with multi path channel, the receiver will be synchronized on the most powerful path. In the final section of this article an example on real data is studied to complete the theoretical approach.

The problem is to synchronize the received signal with the beginning of the frame. In the work presented in [4], the receiver performed a joint starting time frame detection and a Doppler factor estimation This is based on the assumption that the receiver knows the first OFDM symbol of the frame. That knowledge is then used to exploit the channel coherence in frequency domain. So, the method consists in maximizing the coherence while its maximum peak gives the delay and the Doppler factor. Nonetheless, during sea trials realized in 2015, this approach shown its limits in some cases (e.g. a sudden increase in noise power during reception of a frame leads to a poor estimation of Doppler Shift or delay).

\section{Detection Theory}

\section{A. Matched Filter Bank}

Instead of the approach developed in [4], the knowledge of the receiver of the first OFDM symbol of the frame used as a pilot is taken into account. As developed in [5], a maximum likelihood (ML) approach is chosen. Let us define the test (4) to develop the ML method:

$$
\mathcal{H}:\left\{\begin{array}{l}
\mathcal{H}_{0} \rightarrow y(t, D)=w(t), \\
\mathcal{H}_{1} \rightarrow y(t, D)=s\left(D\left(t-t_{0}\right)\right)+w(t) .
\end{array}\right.
$$

As $w(t)$ follows a normal distribution the maximum likelihood algorithm is expressed as:

$$
L(\hat{\Phi})=\max _{\tilde{\phi}}\left[-\frac{1}{\sigma_{w}^{2}} \int_{-\infty}^{\infty}|y(t, D)-s(t, \tilde{\phi})|^{2} d t\right]
$$

where $\tilde{\phi}=\left\{\tilde{t_{0}}, \tilde{D}\right\}$ is the set of every parameters and $\hat{\Phi}$ is the set of estimated parameters. Deriving (5) conducts to $(6)$ :

$$
\Lambda(\hat{\Phi})=\max _{\tilde{\phi}}\left[\Re e\left\{R\left(\hat{t_{0}}, \hat{D}\right)\right\}\right]
$$

where $R\left(\hat{t_{0}}, \hat{D}\right)$ and $s_{0}\left(\hat{t_{0}}, \hat{D}\right)$ are given by:

$$
\begin{gathered}
R\left(\hat{t_{0}}, \hat{D}\right)=\int_{-\infty}^{\infty} s_{0}\left(D\left(t-t_{0}\right)\right) s_{0}^{*}\left(\hat{D}\left(t-\hat{t_{0}}\right)\right) d t \\
s_{0}\left(\hat{t_{0}}, \hat{D}\right)=\sum_{n=0}^{N-1} d_{0, n} g\left(\hat{D}\left(t-\hat{t_{0}}\right)-k T\right) e^{\jmath 2 \pi f_{n} \hat{D}\left(t-\hat{t_{0}}\right)} .
\end{gathered}
$$

The maximization of $L(\hat{\Phi})$ conducts to a joint estimation of delay and Doppler.

One may notice that the ML method will produce good detection results as it maximizes the signal to noise ratio (SNR) thanks to the partial signal knowledge. In addition, the detection reliability could be improved with the pilot sequence length. The greater $N$, the better the detection but assume time invariant of this duration. The choice of the filter $g(t)$ also improves the detection criterion to have a good resolution in Doppler spread. As represented in Fig.1, the detection peak which coordinates are $(-0.1 ; 3)$ gives a precise estimation of the channel parameters that are $t_{0}=0.1 \mathrm{~s}$ and $\nu_{0}=3 \mathrm{~m} / \mathrm{s}$.

In practice, the parameter estimation is realized thanks to a bank of Doppler-shifted OFDM pilot symbol $s_{0}\left(\hat{t_{0}}, \hat{D}\right)$. To ensure a correct detection the Doppler shift step size of the filter bank has to be small enough. It has to be chosen accordingly to the shaping filter $g(t)$. With the chosen configuration in the Fig.1 example, it means a Doppler resolution of $0.2 \mathrm{~m} / \mathrm{s}$, which corresponds to a bank of 150 different filters to compensate a speed between $\pm 15 \mathrm{~m} / \mathrm{s}$. This resolution allows to digitize at least a value close to 95\% of the maximum. This algorithm can be used with a circular buffer or as a block processing. For 


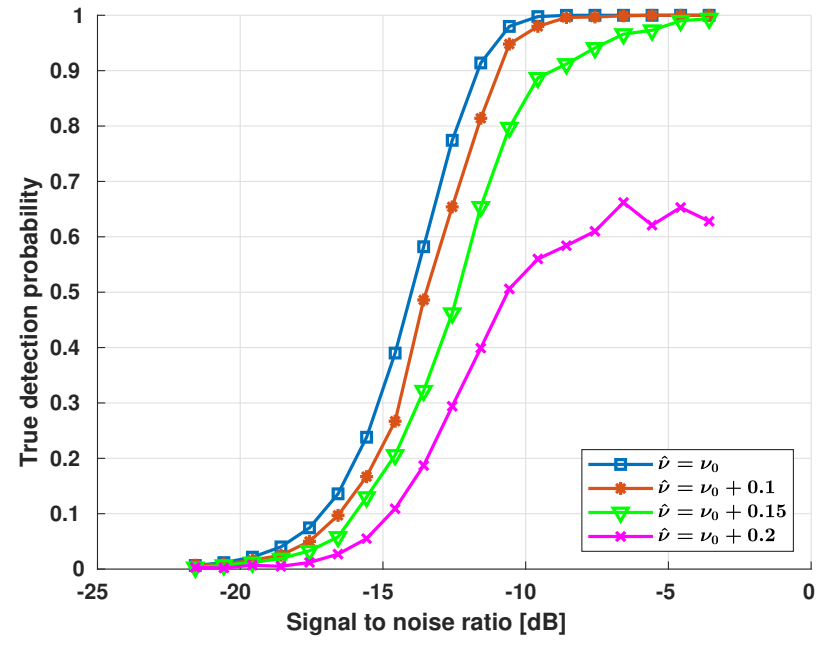

Fig. 2. True detection probability for several Doppler quantification error, with $N=240$ and $\alpha=0.8$

the present article the block processing approach is chosen, with blocks of duration $T$.

To make the receiver able to go in decoding mode, a peak detection has to be performed on the test in (4). To perform this detection a threshold has to be define. Let now assume that the noise variance as known to study the effect of filter bank resolution on detection. Obviously, this assumption is far from real trials but is used here as a reference of the 'best detector'. In Fig.2, the influence of a Doppler estimation error due to the filter bank resolution is shown. When the error on the estimation increases, the true detection probability is shifted to the right. The gap with the perfect estimation becomes larger when the Doppler speed error increases. If the error becomes too important $( \pm 0.2 \mathrm{~m} / \mathrm{s})$, the peak may not be sampled correctly. It indicates that the step size is too small. As the error comes to $0.5 \mathrm{~m} / \mathrm{s}$, the number of true detection drops drastically. This test shows that to have a rough estimate of the Doppler speed the filter bank resolution must be much less than $1 \mathrm{~m} / \mathrm{s}$ with $N=240$ and $\alpha=0.8$.

As defined in [9], for the test in (4) the optimal detection threshold is

$$
\Gamma(k)=\sqrt{\sigma_{w}^{2}(k)} Q^{-1}\left(P_{f a}\right) .
$$

In (9), the function $Q^{-1}(x)$ stands for the tail distribution of the inverse standard normal distribution. A new threshold $\Gamma(k)$ has to be calculated for each

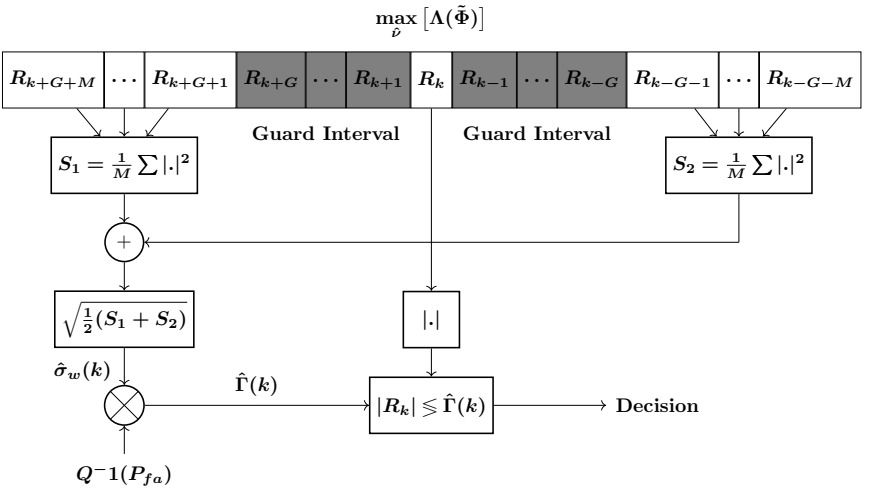

Fig. 3. CA-CFAR detector for a complex input signal with an additional unknown phase

block $k$. It also requires the definition of a false alarm probability $P_{f a}$ and the noise power $\sigma_{w}^{2}(k)$ knowledge. In practice the noise power is unknown, which implies that a noise power estimation has to be made. To make the threshold operation adaptive to the received signal range, a cell averaging constant false alarm rate (CACFAR) algorithm is used, as described in [7].

\section{B. CA-CFAR Detector}

Let now make a short development of CA-CFAR parameters tuning. The main principle is as described in Fig.3. First take the maximum filter bank output and store the filter output (for a particular speed) that gave the maximum. The noise power $\hat{\sigma}_{w}^{2}(k)$ is estimated over $2(M-G)$ values thanks to samples of each part of the peak. Here $G$ is the size of 'guard intervals', that are the closest values of the maximum. The detection threshold is determined based on the $P_{f a}$, and the test is performed. So, the issue is to determine the $M, G$ and $P_{f a}$ values.

The number of samples in the guard interval depends on the peak resolution in function of the delay. To exclude values too close to the maximum, one has to choose $G$ accordingly. With the modem parameters, $G$ equals $0.2 \mathrm{~ms}$ excludes the main lobe and secondaries lobes. Let now determine the value of $M$. Due to the correlation, the delay range is in the interval $[-T ; T]$ with $T$ the OFDM symbol period plus cyclic prefix. To minimize the variance bias of estimation, $M$ could be set at $M=T-G$. The last parameter to tune is the false alarm probability (that belongs to $] 0 ; 1[$ ). This parameter allows the modem to be more sensitive to the noise level. However, if the $P_{f a}$ is too high the 


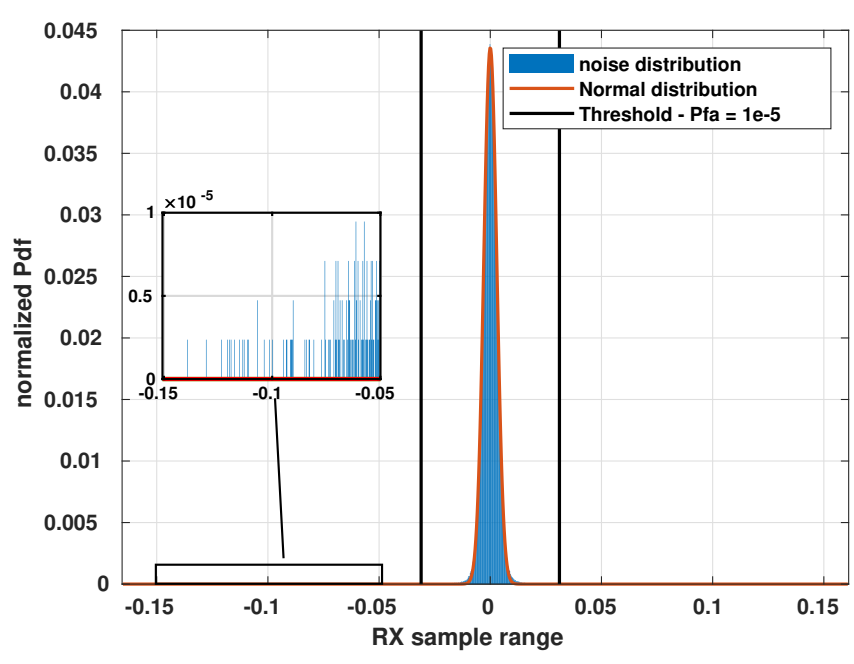

Fig. 4. Noise distribution - record length of 4 seconds

modem goes to decoding mode for an entire frame duration. So, the risk is to miss a frame arriving during this time laps. A low $P_{f a}$ is less sensitive to noise level, but conduct to miss received signals in low SNR. The best trade-off is to set a low $P_{f a}$, ensuring that the passage to decoding mode is the safest. Also, a frame detection in a low SNR could only conduct to a high bit error rate, which has no interest.

\section{Experimental Conditions}

During sea trials the modem described in [4] is used. The distance between the transmitter and receiver is about $800 \mathrm{~m}$, and 20 frames were transmitted in a row. A frame is composed by a symbol pilot and 24 OFDM symbols of $T_{0}=0.06 \mathrm{~s}$. The CP length $\tau$ is set at $0.02 \mathrm{~s}$, so that the entire frame duration is 2 seconds. Each frame is send within a period of $1.5 \mathrm{~s}$ after the previous one. The signal bandwidth is located in the $8-12 \mathrm{kHz}$, and $N$ the number of subcarrier is set at 240 .

\section{SEa Records}

The problematic is now to choose the false alarm probability $P_{f a}$ for sea trials experiments. In Fig.4, the noise distribution measured during 4 seconds is represented. This distribution is representative for the considered experiment described in the previous section. As can be noticed, a large part of noise samples follows a normal distribution. So, setting the $P_{f a}$ at $10^{-5}$ excludes efficiently noise samples to be detected after the matched filter bank. For this record length, the corresponding detection threshold is represented in Fig. 4 by vertical black plots.

Though, the noise also has a impulse components as can be seen in the magnified part of Fig.4. Such samples creates peaks that can't be discarded by the detection threshold as defined above. So, the false alarm probability has to be low enough to take the impulsive noise component into account. This value should be adjusted according to sea conditions. A larger value fits for degraded transmission, for example when the wind force is important creating waves and bubbles into water.

As can be seen in Table I, a threshold calculated with a false alarm probability of $10^{-5}$ results in many false detection. It means that the threshold is too low, which results in detecting some of the impulsive noise components. After each detection the modem is in decoding mode during 2 seconds. But, no communication signal is received in the case of a bad detection. Hence, if a frame was received during the 2 seconds after a bad detection, the message could hardly be decoded as the modem is neither able to synchronize nor compensate for the Doppler shift. In the best case, only a partial decoding of the frame could be performed. On the other hand, when the false alarm probability is well defined (e.g. $10^{-12}$ ), only real frames are detected. In this example, there were no error during the transmission, which underline the threshold value importance.

TABLE I

FALSE ALARM PROBABILITY INFLUENCE ON RECEIVER DECODING ACCURACY IN THE CONTEXT OF SEA TRIALS

\begin{tabular}{|c|c|c|c|}
\hline $\boldsymbol{P}_{\boldsymbol{f a}}$ & $\boldsymbol{N}_{\text {bad detections }}$ & $\boldsymbol{N}_{\text {sent frames }}$ & BER \\
\hline $10^{-5}$ & 17 & 20 & 0.2504 \\
\hline $10^{-12}$ & 0 & 20 & 0 \\
\hline
\end{tabular}

\section{CONCLUSION}

In this article a methodology to choose the filter bank parameters was presented. The filter that gave the maximum correlation is feed to the adaptive detector. An analysis with the CA-CFAR detector parameter tuning was realized. This work is supported by sea tests, that showed that the theoretical approach considering a normal distribution of noise is too simplistic. So, the false alarm probability has to be lowered in order to ensure a few false detection in 
absence of signal. Main benefits of the matched filter bank coupled with a CA-CFAR threshold are a faster treatment and a better estimation, which significantly decrease the bit error rate.

\section{ACKNOWLEDGMENT}

This work was funded in the scope of the NEMOSENS project (see [3]). The authors would like to thank all NEMOSENS partners and specifically RTsys for their valuable help during sea trial. NEMOSENS is an officialy recognized project of the global economic competitiveness "Pôle Mer Bretagne", funded by BPI France and Region Bretagne. RTsys is the work package leader with the partnership of FMC, ENSTA Bretagne and IMT-Atlantique.

\section{REFERENCES}

[1] R. Masiero, P. Casari, and M. Zorzi, "The nautilus project: Physical parameters, architectures and network scenarios," in OCEANS'11 MTS/IEEE KONA, Sep. 2011, pp. 1-10.

[2] J. Kalwa, "The racun-project: Robust acoustic communications in underwater networks - an overview," in OCEANS 2011 IEEE - Spain, June 2011, pp. 1-6.

[3] Pôle Mer Bretagne Atlantique, "NEMOSENS Project Goals," https://www.pole-mer-bretagne-atlantique.com/ $\mathrm{fr} /$ securite-et-surete-maritimes/project/nemosens, 2019, online; accessed 20 March 2019.

[4] A. Bourré, S. Lmai, C. Laot, and S. Houcke, "A robust ofdm modem for underwater acoustic communications," in 2013 MTS/IEEE OCEANS - Bergen, June 2013, pp. 1-5.

[5] B. S. Sharif, J. Neasham, O. R. Hinton, and A. E. Adams, "A computationally efficient doppler compensation system for underwater acoustic communications," IEEE Journal of Oceanic Engineering, vol. 25, no. 1, pp. 52-61, Jan 2000.

[6] S. F. Mason, C. R. Berger, S. Zhou, and P. Willett, "Detection, synchronization, and doppler scale estimation with multicarrier waveforms in underwater acoustic communication," IEEE Journal on Selected Areas in Communications, vol. 26, no. 9, pp. 1638-1649, December 2008.

[7] H. Rohling, "Radar cfar thresholding in clutter and multiple target situations," IEEE Transactions on Aerospace and Electronic Systems, vol. AES-19, no. 4, pp. 608-621, July 1983.

[8] S. Lmai, A. Bourré, C. Laot, and S. Houcke, "Advantages of pulse-shaping applied to ofdm systems over underwater acoustic channels," in 2012 Oceans, Oct 2012, pp. 1-7.

[9] S. M. Kay, Fundamentals Of Statistical Processing, Volume 2: Detection Theory, ser. Prentice-Hall signal processing series. Pearson Education, 2009. [Online]. Available: https://books.google.fr/books?id=wwmnY9xyt9MC 